ISSN: 2386-3919 - e-ISSN: 2386-3927

DOI: https://doi.org/10.14201/et2020381728

\title{
MINECRAFT EN EL APRENDIZAJE DE HABILIDADES SOCIALES PARA PERSONAS CON TRASTORNO DEL ESPECTRO DEL AUTISMO
}

\section{Minecraft in the Learning of Social Skills for People with Autism Spectrum Disorders}

\author{
Mayca Villén DE ARRIBAS
}

Recibido: 18/01/2020; Aceptado: 16/09/2020; Publicado: 30/12/2020

Ref. Bibl. MAYCA VILLÉN DE ARRIBAS. Minecraft en el aprendizaje de habilidades sociales para personas con trastorno del espectro del autismo. Enseñanza $\mathcal{E}$ Teaching, 38, 1-2020, 7-28.

RESUMEN: Los videojuegos, hoy en día, suponen la forma de entretenimiento más extendida de la sociedad actual. Partir de este interés general para elaborar nuevas terapias y nuevos enfoques que aprovechen al máximo nuestras capacidades, por diferentes que sean, es la base de esta investigación.

Este artículo presenta una descripción científica y analiza la mejora de las habilidades sociales que presentan un grupo de personas con trastorno del espectro del autismo gracias a la creación de un club basado en el videojuego Minecraft. Los participantes fueron 27 usuarios y sus familiares, con edades comprendidas entre los 8 y los 20 años. Utilizamos un diseño cuasiexperimental con metodología cualitativa. El desarrollo se ha basado en tres dimensiones e indicadores atendiendo a diferentes instrumentos. Para analizar los objetivos de las dimensiones aplicamos la prueba de Wilcoxon a los test presentados a los socios del club, análisis de las interacciones y grupo de discusión de los profesionales que han intervenido en el estudio, además del análisis del Cuestionario de Evaluación sobre el Club Minecraft (CECM), que ha contado con la participación de las familias.

Concluimos que los usuarios muestran una motivación por el videojuego que favorece el aprendizaje de habilidades nuevas, además de ser divertido, por lo que se demuestra que el uso de Minecraft presenta una eficacia terapéutica para el aprendizaje de habilidades sociales en personas con TEA. 
Palabras clave: Minecraft; aprendizaje; autismo; innovación.

SUMMARY: Video games today, represent the form of entertainment of today's society. From this general interest to develop new therapies and new approaches that make the most of our capabilities, however different they are the basis of this research.

This article presents a scientific description and analyzes the improvement of social skills presented by a group of people with Autism Spectrum Disorder thanks to the creation of a club based on the video game Minecraft. The participants were 26 users and relatives, aged between 8 and 20 years. We use a quasi-experimental design with qualitative methodology. The development has been based on three dimensions and indicators with different instruments. In order to analyze the objectives of the dimensions we applied the Wilcoxon test to the tests presented to the club members, analysis of the interactions and discussion group of the professionals who participated in the study, in addition to the analysis of the Evaluation Questionnaire on the Club Minecraft (CECM) with the participation of families.

We conclude that the users show a motivation for the video game that favors the learning of new skills, in addition to being fun, so, it is demonstrated that the use of Minecraft presents a therapeutic efficacy for learning social skills in people with ASD.

Key words: Minecraft; learning; autism; Innovation.

\section{INTRODUCCIÓN}

Siguiendo la definición de la quinta edición del Diagnostic and Statistic Manual of Mental Disorders (Manual Diagnóstico y Estadístico de Trastornos Mentales, más conocido como DSM-5), publicado por la American Psychiatric Association (APA), las personas con Trastorno del Espectro del Autismo (TEA) pueden presentar deficiencias en la comunicación social, tales como responder de manera inapropiada en las conversaciones, malinterpretar interacciones no verbales, o tener dificultades en la construcción de relaciones de amistad adecuadas a su edad. Además, las personas con TEA presentan comportamientos restringidos y repetitivos como ser altamente dependientes de las rutinas, muy sensibles a los cambios de su medio ambiente, o se centran intensamente en intereses inapropiados, todo esto teniendo siempre en cuenta que los síntomas de las personas con TEA implican la idea de un continuo de dimensiones del desarrollo alteradas, con algunas personas que muestran síntomas leves y otros que pueden presentar síntomas más severos. Para el desarrollo de esta investigación se ha elegido el videojuego Minecraft a través de la creación de un club de usuarios en el centro de Desarrollo Autismo Albacete, una asociación que da respuesta a las necesidades de las personas con TEA y sus familiares en Albacete. Este videojuego de Minecraft fue originariamente creado por Markus Persson, también conocido como Notch, que es además el nombre que recibe su personaje dentro de la plataforma de juego. Minecraft ha 
MINECRAFT EN EL APRENDIZAJE DE HABILIDADES SOCIALES PARA PERSONAS CON TRASTORNO DEL ESPECTRO DEL AUTISMO

demostrado ser muy popular desde su lanzamiento en 2009, con más de 176 millones de copias vendidas, en el conjunto de todas las consolas y dispositivos para los que está disponible, según su cuenta de Twitter en 2019 (@Minecraft), convirtiéndose así en el videojuego más vendido de la historia.

Minecraft es un videojuego independiente de construcción, basado en la libertad de acción y en la creación de todo lo necesario para avanzar y transformar un mundo virtual. Este tipo de videojuegos se denominan sandbox.

\section{Videojuegos Y AUTISMO}

Actualmente no existe ningún trabajo previo sobre Minecraft y TEA anterior a este estudio donde se haya abordado el tema de los videojuegos como terapia para personas con TEA. Por tanto, el análisis de las investigaciones relacionadas con este ámbito se ha basado, por una parte, en las publicaciones fundamentadas en el trastorno del espectro del autismo (TEA) y tecnologías de la información y la comunicación (TIC), donde se incluyen dispositivos, software o videojuegos afines a esta línea de estudio, para, de esta forma, ir avanzando en los trabajos sobre aprendizaje basado en videojuegos (Game Based Learning) acordes a la temática del aprendizaje en habilidades sociales y así terminar incluyendo los estudios desarrollados específicamente sobre el videojuego Minecraft en el ámbito educativo.

En el estudio realizado por Lozano-Martínez y Alcaraz-García (2011), Software educativo para la enseñanza de competencias emocionales en alumnado con Trastornos del Espectro Autista, se presenta el Trastorno desde una perspectiva ligada a las terapias y herramientas tecnológicas porque los encuentran atrayentes debido a sus cualidades visuales y a la posibilidad de repetir fácilmente sus acciones favoritas.

El artículo «The Intelligent Robot Contents for Children with Speech-Language Disorder» (Lee y Hyun, 2015) hace referencia al desarrollo de un programa de intervención a través de un robot en niños/as con trastornos del lenguaje oral asociados a trastorno del espectro autista (TEA) a través de la interacción lingüística. Además, analiza los datos de diagnóstico y evaluación. Este estudio se realiza desde un enfoque educativo entretenido y desde el lenguaje práctico y es por ello que lo he elegido para mi estudio, ya que los resultados indican que los niños con autismo mejoraron en las conversaciones e intercambios emocionales de expresiones. Por tanto, concluyen que los niños con discapacidad tienen dificultades para interactuar con la gente debido a que los seres humanos utilizamos complicadas señales lingüísticas.

Aresti-Bartolome y Garcia-Zapirain (2014) nos presentan Technologies as support tools for persons with autistic spectrum disorder: a systematic review. En este estudio se analizan las aplicaciones más relevantes y las tecnologías desarrolladas desde 2004 hasta 2013, y que más ampliamente suelen usarse para trabajar las áreas afectadas por el trastorno del espectro del autismo: comunicación e interacción, aprendizaje social y habilidades de imitación, y condiciones asociadas al 
trastorno. Las conclusiones acuerdan que la tecnología sirve como herramienta clave para personas con TEA, sus familias o profesionales. Sin embargo, es esencial ajustar el contenido a la edad de los niños y limitar el tiempo de uso.

En cuanto al estudio realizado por Molyneux, Vasudevan y Gil de Zúñiga (2015), Gaming Social Capital: Exploring Civic Value in Multiplayer Video Game, supone que los jugadores que desarrollan lazos y trabajan juntos con una comunidad de compañeros van construyendo el capital social del juego, el propio sentido de pertenencia y la participación en una comunidad de jugadores. Este tema está muy relacionado con mi línea de investigación, que trata de desarrollar las habilidades sociales en niños/as con TEA en un ambiente de juego interactivo.

Una de las principales aportaciones de este artículo que más valoro para mi estudio es la creación de una medida de capital social de juego. Este concepto capta las conexiones e interacciones entre los jugadores en un ambiente de multijugador que producen un sentido de pertenencia a una comunidad común a todos ellos, factor muy relevante a tener en cuenta en la adquisición de habilidades sociales en entornos multimedia.

Otra investigación acorde a esta línea de serious games es The effect of the video game Mindlight on anxiety symptoms in children with an Autism Spectrum Disorder (Wijnhoven, Creemers, Engels y Granic, 2015). El videojuego Mindlight se concluye como una terapia eficaz, lo que proporciona una contribución significativa basada en la evidencia en el tratamiento de la ansiedad en los niños con TEA.

La investigación de Ferguson, Gillis y Sevlever (2013), titulada "A Brief Group Intervention Using Video Games to Teach Sportsmanship Skills to Children with Autism Spectrum Disorders", presenta el estudio sobre las dificultades en habilidades sociales, que representan un déficit fundamental para los niños con trastornos del espectro autista (TEA). Los resultados sugieren que este programa se puede utilizar con eficacia para enseñar habilidades de deportividad a los niños con TEA. También se discute la utilización de la tecnología de videojuego como parte de las habilidades sociales de programas de intervención.

Siguiendo en la dirección de investigaciones acerca de videojuegos y sus aplicaciones educativas o terapéuticas, nos encontramos con el artículo de del Moral y Fernández García (2015), Videojuegos en las aulas: implicaciones de una innovación disruptiva para desarrollar las Inteligencias Múltiples, donde nos muestran los videojuegos como una innovación que requiere necesariamente de los recursos tecnológicos adecuados para llevarla a cabo, aunque, a la vista de sus valoraciones, se ha hecho patente la precariedad de las infraestructuras de sus centros escolares.

Otra investigación publicada sobre serious games es la de Méndez y Del Moral (2015) titulada "Investigación e innovación educativa con videojuegos». Uno de los puntos ha sido el tratamiento de los videojuegos en su relación con las normas como propio juego. La mayoría de los videojuegos poseen reglas al igual que los juegos tradicionales, sin embargo, existen diferencias sustanciales dado que los jugadores pueden intervenir e influir en las consecuencias derivadas del mismo, llegando incluso a involucrarse a nivel emocional. 
Todos coinciden en la necesidad de seleccionar adecuadamente los videojuegos en sintonía con los objetivos que se quieren alcanzar, sistematizando las intervenciones para controlar sus efectos, conscientes de su carácter disruptivo (Del Moral y Fernández, 2015). En todos los casos, se apuesta por rentabilizar el compromiso (engagement), es decir, su capacidad para atraer la atención de los jugadores, lograr sumergirlos en las tareas propuestas y transformarlos en entornos de aprendizaje.

En cuanto a estudios sobre Minecraft enfocados al uso del videojuego en contextos educativos, nos encontramos el trabajo de Short (2012), basado en la enseñanza de las ciencias a través de un mundo virtual de Minecraft, en el que se concluye que Minecraft puede ser utilizado por el profesor en las distintas lecciones como una herramienta de trabajo más. La exposición a conceptos científicos a través de este videojuego tendría un efecto positivo en los estudiantes que, a su vez, pueden relacionarlos con las experiencias de la vida real.

En el ámbito español, destaca el estudio realizado por Sáez y Domínguez (2014) fundamentado en la integración pedagógica de la aplicación Minecraft Edu en Educación Primaria donde se concluye que, a pesar de no encontrar ventajas significativas, mejora la creatividad, desarrolla el descubrimiento, es divertido y aplica de un modo efectivo contenidos orientados a edificios históricos.

\section{Club Minecraft Desarrollo Autismo Albacete}

\subsection{Asociación Desarrollo Autismo Albacete}

La Asociación Desarrollo Autismo Albacete apuesta por la utilización de medios tecnológicos en general y de la informática en particular como herramienta educativa, basándose en la persona y no en la tecnología disponible, dejando de este modo abierta la opción de que este apoyo sea directamente orientado a la persona con trastorno del espectro autista y/o de forma indirecta para aquellos que trabajan con esa persona.

Desde esta perspectiva y desde el interés mostrado por los usuarios de la asociación hacia el videojuego Minecraft, surgió el Club Minecraft de Desarrollo. Las actividades de aprendizaje de este proyecto corresponden a estrategias de aprendizaje diversas que faciliten al usuario la percepción clara de lo que se debe hacer y la respuesta y/o consejo inmediato a sus actos a través del uso de normas. Estas normas establecidas para el club se complementan con los comentarios de los usuarios y la evaluación de los educadores en las asambleas propuestas. 


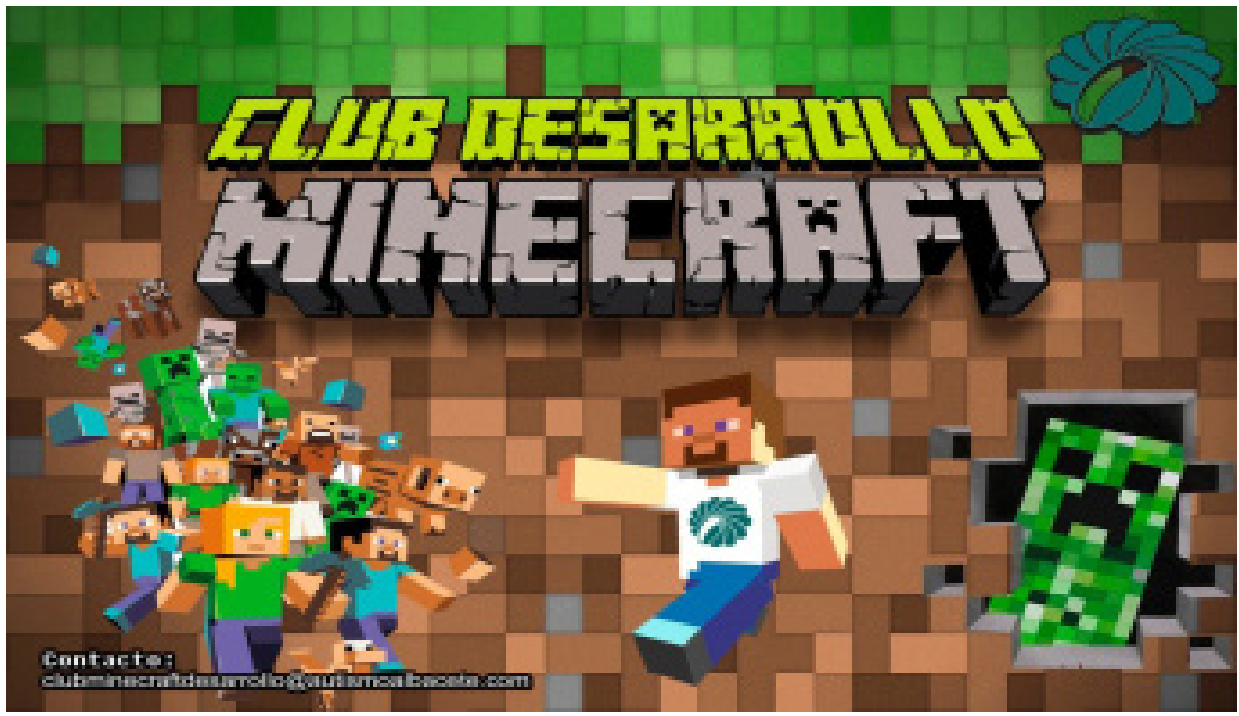

\subsection{Intervención específica del Club Minecraft para personas con TEA}

La metodología en la que se fundamenta este club se basa en la metodología propia en el trabajo con personas con TEA y en concreto para este club se desarrolla a través de asambleas presenciales y agendas, además del uso de referentes visuales que sirvan como apoyo para sus aprendizajes.

Estas asambleas suponen una reunión mensual junto con los otros compañeros del club y educadores y constituyen el encuentro presencial donde poner en práctica lo trabajado a través de la plataforma del videojuego. En la plataforma del videojuego los usuarios pueden interaccionar con el resto de participantes a través del mundo virtual y de los chats, sin embargo, gracias a las asambleas presenciales, pueden participar e intercambiar impresiones sobre aspectos sucedidos en el juego con el fin de poner de manifiesto comportamientos sociales, además de recursos y estrategias que promueven una participación activa dentro y fuera del videojuego.

Las asambleas constituyen uno de los núcleos principales de la metodología activa y constructivista, donde se establecen cauces de comunicación entre los usuarios y usuarias y los educadores. Al mismo tiempo es una vía de resolución de conflictos, descubrimiento de nuevos conceptos o posibilidades del videojuego, además de ofrecer la posibilidad de interacción con nuevos compañeros del club.

Para realizar la asamblea nos sentamos en semicírculo frente a un panel con un cañón donde se proyecta primero la agenda y el propio escenario de Minecraft 
de forma que todos lo tengamos a la vista tanto la pantalla como al resto de los compañeros.

La asamblea la podemos realizar con diferentes finalidades:

- Comienzo y fin de los momentos del día (saludo y despedida).

- Cambio o paso a la siguiente actividad.

- Revisión de la convivencia en el mundo virtual y resolución de los posibles conflictos que puedan ocurrir.

- Propuestas o sugerencias.

- Acontecimientos específicos: acto de navidad, regalo de camiseta del club...

En estas asambleas se utilizan agendas visuales como medio para presentar la organización de las actividades o momentos clave del día, a través de dibujos esquemáticos acompañados del vocabulario correspondiente y que representan de forma visual cada una de las actividades a realizar. Esta presentación se expone tanto proyectada en la pizarra para poderla comentar de forma grupal como en formato de papel con el fin de que cada uno pueda ir realizando de forma individual la anticipación y conclusión de los diferentes momentos en los que se compone cada encuentro.

Este apoyo visual favorece la mejor interpretación y planificación de la sesión por parte de los usuarios con TEA, proporcionando instrumentos para su estabilidad emocional. Para su elaboración hacemos uso de los pictogramas aportados por ARASAAC, el Portal Aragonés de la Comunicación Aumentativa y Alternativa (http:// arasaac.org).

En cuanto a la metodología de uso de las agendas visuales, se realizan como primera actividad de la asamblea después del ritual de entrada y saludo. Se realiza en presencia del niño/a, al mismo tiempo que se va explicando la secuencia. A lo largo de la actividad se puede consultar para la explicación de cambios de actividad o del paso de una a otra. Esta agenda puede ser consultada en cualquier momento por el niño/a si lo necesita.

Continuando con los métodos de trabajo utilizados nos encontramos con el apoyo conductual positivo basado en un conjunto de procedimientos y técnicas destinadas a cambiar el entorno para hacer que la conducta a extinguir pase a ser irrelevante e inútil a nivel social y, por consiguiente, hacer que la conducta alternativa adecuada sea más funcional.

Entender las motivaciones e, incluso, la necesidad de expresión de la persona a partir de este tipo de conductas a extinguir debe conducirnos a un buen manejo de las mismas, para así poder dotar a la persona de herramientas e instrumentos desde el plano comunicativo al de la gestión emocional.

Bajo esta estructura se plantea el ingreso al club a través de una serie de momentos en los que se trabaja este apoyo conductual positivo.

En un primer momento se hace entrega del material de la asamblea: 
- La firma del contrato con las normas y el carnet, además de un listado de los usuarios del club con su alias y foto para que puedan ser reconocidos $\mathrm{y}$, de esta forma, generalizar en las asambleas los contactos tenidos a través del videojuego. Por una parte, se encuentran las Normas Durante el Juego Online y, por otra, las Normas para las Asambleas, que guían el comportamiento de cada miembro en las dos situaciones de socialización.

- El material del videojuego y unas nociones básicas sobre cómo moverte o jugar en el mundo virtual.

A partir de aquí se pasa a un segundo momento en el que se trabaja desde el videojuego Minecraft directamente con la propia convivencia en el mundo Minecraft y las propuestas de objetivos comunes como forma de trabajo cooperativo dentro del espacio del videojuego.

Siguiendo esta línea, en un tercer momento se aplica la mediación como estrategia, por una parte, en la llegada a acuerdos entre los usuarios y usuarias y, por otra parte, en los conflictos que puedan suceder en el juego y fuera de él, para así poder apoyar la posibilidad de analizar de forma grupal los acontecimientos que surjan y, de esta forma, alcanzar posibles soluciones pacíficas.

Además de todo esto, otro punto importante dentro de estos métodos son las insignias del club, como son las camisetas de cada temporada, los macutos con los apodos de cada usuario o las chapas del club, que pretenden promover la responsabilidad y la estabilidad emocional entre los participantes como miembros del club.

FIGURA 2

Mundo virtual del Club Minecraft preparado para Halloween

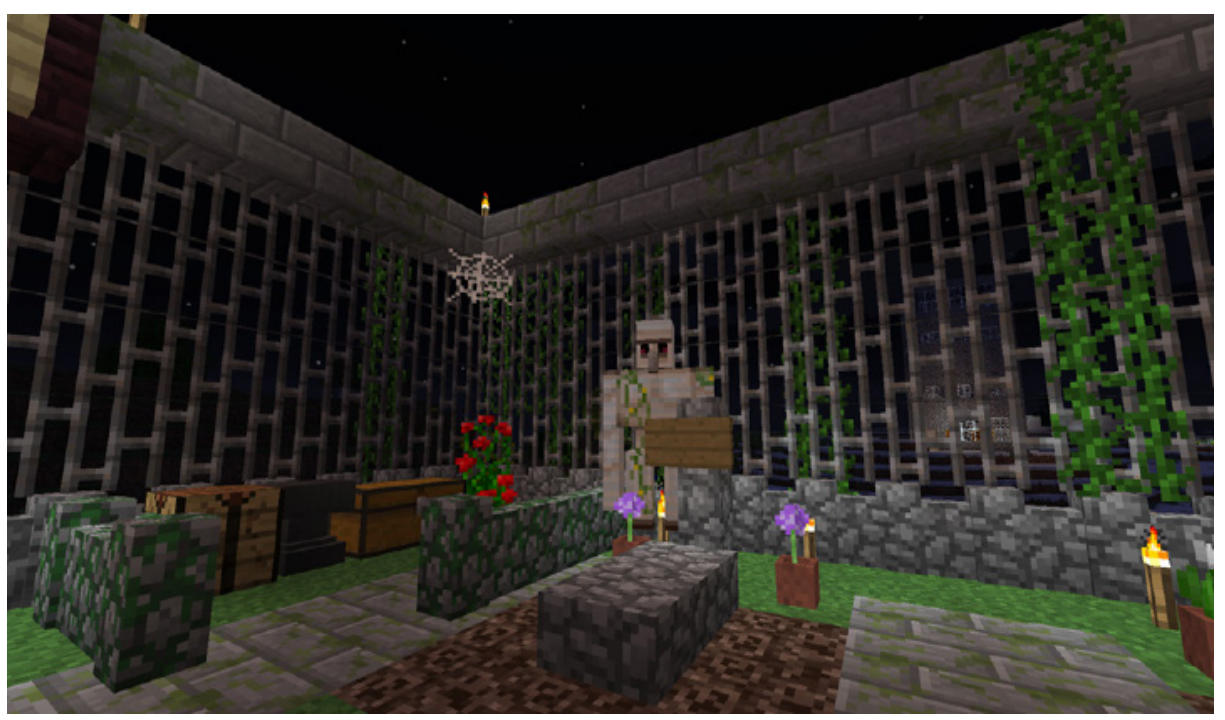


MINECRAFT EN EL APRENDIZAJE DE HABILIDADES SOCIALES PARA PERSONAS CON TRASTORNO DEL ESPECTRO DEL AUTISMO

\section{DiseÑO METODOlÓGICO DE LA INVESTIGACIÓN}

Como objetivo general de este estudio se plantea analizar las ventajas del uso de Minecraft en el aprendizaje de habilidades sociales para personas con TEA. Este objetivo general se concreta en los siguientes objetivos específicos:

- Valorar el grado de motivación para las interacciones sociales de los usuarios con TEA a través del uso de Minecraft.

- Comprobar las interacciones que se producen en contextos virtuales y fuera de ellos.

- Utilizar Minecraft como herramienta de aprendizaje colaborativo.

- Analizar el interés mostrado por las personas con TEA por pertenecer a un club y cumplir las normas establecidas.

- Evaluar las actitudes de los profesionales y familiares frente al uso de un videojuego como terapia con personas con TEA.

Para la realización del estudio me he basado en la investigación cuasiexperimental consistente en la manipulación de una variable experimental no comprobada, en condiciones rigurosamente controladas, con el fin de describir de qué modo se produce esta situación de interacción social en particular en este caso en concreto.

Para esta investigación he llevado a cabo un estudio de caso, por tanto, este tipo de investigación nos permite intervenir y determinar si el videojuego Minecraft sirve como hilo conductor en el aprendizaje y mejora de las habilidades sociales en las personas con TEA y si, a pesar de que cada grupo o persona es único e irrepetible, se pueda replicar en otros contextos de terapia para este colectivo en especial o incluso para otros colectivos con discapacidad o sin ella.

Desde el marco teórico expuesto anteriormente, esta investigación ha llevado a cabo un estudio sobre el uso del videojuego Minecraft para el aprendizaje y mejora de las habilidades sociales en personas con TEA, en el que se analizan los diferentes aspectos y factores inclusivos. Este trabajo se centra en indagar, a lo largo del proceso de investigación-acción, en qué condiciones el uso del videojuego Minecraft facilita estos aprendizajes sociales. Por tanto, nuestro ámbito de investigación y el objetivo principal de este estudio consisten en detectar los factores clave que hacen que el videojuego Minecraft favorezca un proceso de mejora desde un enfoque basado en aspectos sociales.

Partiendo de contextos que destacan actitudes relativas al uso de las tecnologías (Sáez López, 2011; Sáez López, 2012), me he basado en diferentes estudios (Sáez López y Domínguez, 2013; Sáez-López, Miller, Vázquez-Cano y DomínguezGarrido, 2015) donde se destacan aportaciones significativamente positivas respecto a la motivación y compromiso de los estudiantes en el uso de Minecraft, para la elaboración de las diferentes dimensiones de la investigación e indicadores propuestos en este estudio, que mantienen una estructura y diseño similar atendiendo a diferentes instrumentos en cada una de dichas dimensiones. 
Estas dimensiones se analizan con instrumentos específicos para, a través de una triangulación de datos, poder asegurar que hay suficiente evidencia para afianzar la validez y se permite minimizar la varianza de error.

Estas dimensiones están indicadas en la siguiente tabla:

TABLA 1

Dimensiones, indicadores e instrumentos

\begin{tabular}{|c|c|c|}
\hline DIMENSIONES & INDICADORES & INSTRUMENTOS \\
\hline \multirow{5}{*}{$\begin{array}{l}\text { Dimensión 1: } \\
\text { Análisis de los } \\
\text { intereses por } \\
\text { Minecraft de las } \\
\text { personas con TEA }\end{array}$} & Motivación de los usuarios & \multirow{5}{*}{$\begin{array}{r}\text { Cuestionario } \\
\text { pretest - postest } \\
\text { Test de Wilcoxon }\end{array}$} \\
\hline & Enfoque lúdico & \\
\hline & Inclusión digital & \\
\hline & Compromiso del usuario & \\
\hline & Interacciones y comunicación & \\
\hline \multirow{4}{*}{$\begin{array}{l}\text { Dimensión 2: } \\
\text { Conclusiones de los } \\
\text { profesionales frente } \\
\text { al uso de Minecraft } \\
\text { como terapia para } \\
\text { personas con TEA }\end{array}$} & Creatividad & \multirow{4}{*}{$\begin{array}{l}\text { Entrevista grupal } \\
\text { (HyperResearch) }\end{array}$} \\
\hline & Estrategias de enseñanza alternativa & \\
\hline & Atención conjunta & \\
\hline & Eficacia terapéutica & \\
\hline \multirow{4}{*}{$\begin{array}{l}\text { Dimensión 3: } \\
\text { Actitudes de las } \\
\text { familias respecto } \\
\text { al Club Minecraft } \\
\text { como terapia }\end{array}$} & Trabajo colaborativo & \multirow{4}{*}{$\begin{array}{r}\text { Cuestionario de } \\
\text { Evaluación sobre el } \\
\text { Club Minecraft (CECM) }\end{array}$} \\
\hline & Adquisición de normas & \\
\hline & $\begin{array}{l}\text { Generalización del mundo } \\
\text { virtual al mundo real }\end{array}$ & \\
\hline & Eficacia y adecuación terapéutica & \\
\hline
\end{tabular}

Para el estudio me he basado en una muestra no probabilística ni aleatoria, de 13 usuarios para el estudio de caso de la dimensión 1, además de los 4 educadores que formamos el club y que hemos realizado la entrevista grupal para la dimensión 2 y tendremos la muestra de 14 familiares que han participado en la elaboración del cuestionario de la dimensión 3.

\subsection{Dimensión 1: Análisis de los intereses por Minecraft de las personas con TEA}

Al encontrarnos con un diseño cuasiexperimental no aleatorio, se necesita de un pretest para reducir la parcialidad. Por ello, en esta dimensión se ha realizado un cuestionario dicotómico de pretest/postest para los usuarios del Club Minecraft, que se encuentran únicamente ante dos alternativas: responder afirmativa o 
MINECRAFT EN EL APRENDIZAJE DE HABILIDADES SOCIALES PARA PERSONAS CON TRASTORNO DEL ESPECTRO DEL AUTISMO

negativamente (Respuesta Sí/No), permitiendo la obtención de cálculos estadísticos a partir del conjunto de respuestas del grupo seleccionado.

Este pretest es una encuesta que se realiza antes del inicio del Club Minecraft y el postest es la misma encuesta pero posterior al inicio del club. Se realizan para verificar el efecto del club en las actitudes sociales de los usuarios inscritos.

Por tanto, es una prueba no paramétrica de comparación de dos muestras relacionadas, es libre de curva, no necesita una distribución específica. Presenta un nivel ordinal de la variable dependiente utilizándose para comparar dos mediciones de rangos (medianas) y determinar que la diferencia no se deba al azar y sea estadísticamente significativa.

En la elaboración de este instrumento se han tenido en cuenta tanto la redacción del contenido como la tipografía del cuestionario y la duración breve del mismo, además de la realización in situ, para que se puedan percibir más claramente las dificultades del instrumento si las hubiera.

\subsection{Dimensión 2: Conclusiones de los profesionales frente al uso de Minecraft como terapia para personas con TEA}

Esta dimensión se basa en las conclusiones de los profesionales frente al uso de Minecraft como terapia para personas con TEA y es por ello que la elección de un instrumento de recogida de datos para esta dimensión obedece a una serie de criterios no arbitrarios y justificables desde el punto de vista de la metodología científica, por lo que he seleccionado la creación de una entrevista grupal como herramienta de recogida de información. Para facilitar su puesta en marcha, las entrevistas cualitativas deben ser abiertas, de tal forma que los participantes puedan expresar sus experiencias.

Al estar esta investigación relacionada con profesionales en las terapias para personas con TEA, es conveniente que los participantes sean representativos de la población estudiada, por tanto, los perfiles de los integrantes del grupo son:

- Psicóloga (coordinadora del proyecto).

- Educadora de las terapias grupales e individuales que se aplican con ayuda de herramientas TIC.

- Educador del piso tutelado.

- Maestra de Educación Infantil y psicopedagoga (voluntaria del proyecto e investigadora).

Mediante esta heterogeneidad estaría asegurando el equilibrio y la representación de los distintos ámbitos en los que se aplican las terapias con personas con TEA. 


\subsection{Dimensión 3: Actitudes de las familias respecto al Club Minecraft como terapia}

En esta tercera dimensión se encuentra el Cuestionario de Evaluación sobre el Club Minecraft (CECM), dirigido a las familias de los usuarios del club, con el fin de analizar sus impresiones y actitudes frente al Club Minecraft como herramienta terapéutica.

Este cuestionario consta de 7 preguntas en total, de las cuales 6 son preguntas cerradas mediante una ESCALA DE LIKERT, ofreciendo una afirmación en la que se pide que se califique del 0 al 4 según su grado de acuerdo con la misma, siendo el 1 totalmente en desacuerdo y 4 totalmente de acuerdo, con el fin de evitar afirmaciones neutras y 1 última pregunta de elección múltiple, donde se pueden elegir una o varias opciones o apuntar otra que se crea oportuna.

Este cuestionario ha sido realizado por 14 familiares, que han tenido la opción de completarlo en formato online o en papel en las asambleas.

FIGURA 3

Aspecto del cuestionario online

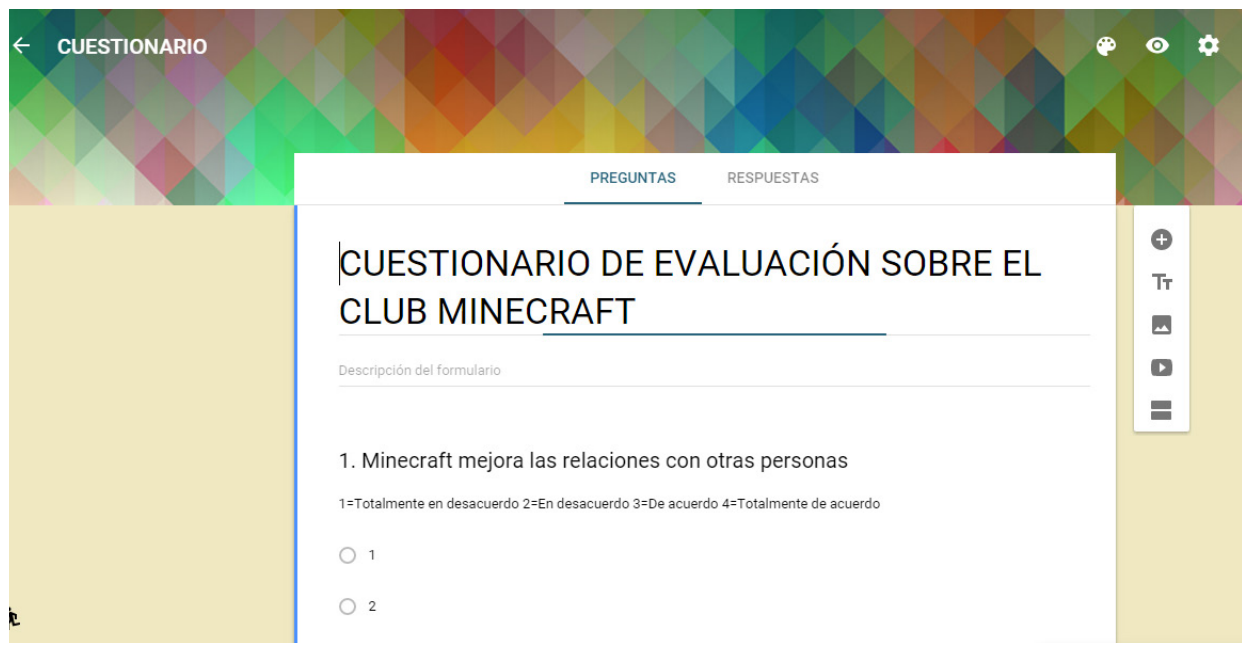

\section{Resultados}

Entre las dificultades encontradas para este proyecto, la primera y fundamental ha sido el número de sujetos para la muestra, puesto que, al ser un proyecto nuevo y, además, destinado específicamente a personas con TEA, ha supuesto un condicionante para el estudio. Otra dificultad ha sido el uso de servidores, ya que no aguantan un número elevado de usuarios conectados al mismo tiempo. Sin embargo, a pesar de estas dificultades, el proyecto se ha podido realizar sin problemas gracias a la colaboración de todos los usuarios, sus familiares y los 
profesionales del club. Este club seguirá sus actividades programadas para las siguientes temporadas, partiendo siempre de los intereses y necesidades de los usuarios y usuarias del club.

Los resultados obtenidos se clasifican, por tanto, en las siguientes dimensiones:

\subsection{Resultados para la dimensión 1 de la investigación}

En cuanto a las técnicas e instrumentos de recogida de datos utilizados, como ya se ha mencionado en el apartado anterior, han sido un cuestionario mixto que se administra antes (pretest) y otro que se aplica después (postest) de la puesta en marcha del proyecto. Una vez que la información se recoge, se han aplicado un análisis descriptivo, un análisis estadístico y una prueba de Wilcoxon para medir diferencias significativas antes y después de este programa de entrenamiento.

Las actividades desconocidas o nuevas pueden ser un impedimento para la participación de las personas con TEA debido a la intolerancia a la incertidumbre, el no saber lo que va a pasar o las situaciones nuevas que ellos no conocen ya que pueden provocar ciertos niveles ansiedad.

Sin embargo, en esta dimensión se aprecia que el videojuego aumenta la capacidad de adaptación de los jugadores a contextos nuevos, gracias a esta motivación reflejada, ya que se ve reflejado un alto índice de respuestas positivas para ítems relacionados con participar en el juego, hablar de él o las creencias sobre las finalidades del videojuego en cuanto a divertirse y aprender cosas nuevas y crear.

Por tanto, contamos con una motivación inicial y una recepción fantástica por parte de los usuarios que les hace querer participar de forma activa en este club, además, mostrando implicación por su parte para animar a otras personas a formar parte del club, tal y como muestran los resultados de los cuestionarios pretest y que se consigue mantener a lo largo del proceso con los datos mostrados por el postest.

El videojuego Minecraft es bastante motivador para ellos ya que se fijan nuevos retos, nuevas construcciones y nuevas ideas que aportan en las asambleas. La posibilidad de compartirlo con los demás miembros del club les hace leer libros sobre el videojuego, buscar tutoriales en Youtube o informarse más sobre las posibilidades del juego.

Los análisis estadísticos se han elaborado a partir del paquete estadístico informático IBM SPSS Statistics 22, para acceder al valor de la prueba de Wilcoxon, que se basa en los datos proporcionados por el cuestionario pretest y postest, permitiendo así determinar si hay diferencias significativas antes y después de la aplicación del proyecto de innovación del Club Minecraft.

Se ha optado por esta prueba exacta debido a la poca muestra con la que contamos para el estudio.

En el caso del ítem 3 (en el pretest: «Voy a pertenecer al Club Minecraft al menos hasta verano» y en el postest: «Pertenezco al Club Minecraft desde el comienzo") se aprecian valores no significativos aunque se aproximan a ellos, por lo que existe una tendencia a creer que estos resultados se deben a que en un 
primer momento no estaban seguros de su duración en el club y, sin embargo, a medida que ha ido pasando el tiempo se ha desarrollado el sentimiento de pertenencia a un grupo y cierta responsabilidad tanto grupal como individual.

En el ítem 10 (en el pretest y postest: «Los videojuegos son difíciles») sí que se encuentran valores significativos para la prueba de Wilcoxon que muestran que les parecen más difíciles después de conocer las posibilidades de Minecraft y de los videojuegos en general.

Estos ítems destacan por su significatividad en la prueba de Wilcoxon. En esta línea del análisis descriptivo nos encontramos que el resto de ítems corroboran esta motivación de los usuarios del club, que crea un resultado claro para la dimensión 1 donde se pretendía analizar los intereses de las personas con TEA a través de indicadores que quedan expuestos en estos resultados como son la motivación; su enfoque lúdico; la inclusión digital apreciada a través de la participación en el servidor del club, que supone el aprendizaje de competencias digitales; el compromiso de los usuarios, que, como ya se ha comentado, se reconoce en la prueba efectuada donde se aprecia un aumento en la implicación y un afán por conseguir retos y objetivos del club, y las interacciones y comunicación de las preguntas abiertas.

\subsection{Resultados para la dimensión 2 de la investigación}

Para esta segunda dimensión se ha organizado una entrevista grupal con los profesionales que forman el Club Minecraft. Los comentarios han sido analizados con HyperResearch como método para comprobar y extraer los resultados pertinentes.

Se resaltan los conceptos que han sido utilizados durante el desarrollo de la misma: normas, asambleas, videojuego, club, jugar y habilidades.

\section{FIGURA 4}

Frecuencias e HyperResearch

Code
\begin{tabular}{|l|l|l|l|l|l|}
\hline interés común & Total & Mean & Std Dev & Bar Graph \\
\hline normas & 18 & 9 & 12.728 & \\
\hline divertido & 11 & 5.5 & 7.778 & \\
\hline terapia & 8 & 4 & 5.657 & \\
\hline motivación & 8 & 4 & 5.657 & \\
\hline & 5 & 2.5 & 3.536 & \\
\hline
\end{tabular}

Los resultados a los que se ha llegado gracias a esta herramienta para el análisis de datos cualitativos, se han basado en cuatro bloques: por una parte, destacar que el videojuego proporciona un tema de interés común que facilita la participación de los hermanos y las familias, que también se ha puesto de manifiesto con 
otros miembros del club o compañeros de clase, promoviendo a través de este punto de interés un tema para favorecer la comunicación interpersonal.

Otro punto ha sido que las normas de funcionamiento del club son un factor principal para el funcionamiento del mismo, tanto como club como por terapia en la que se interiorizan normas de convivencia y se generalizan al contexto a trabajar. En este punto, las asambleas son el eje principal de este club, donde se ponen en juego las habilidades aprendidas y expuestas a través de las normas.

Otro resultado principal a destacar es que es una terapia con un componente divertido, propio del videojuego, que facilita el trabajo y los aprendizajes gracias a la motivación mostrada por los usuarios.

Además, destaca como resultado el amplio abanico de posibilidades que tiene un club basado en el videojuego Minecraft como terapia, tanto por la creatividad que surge de este videojuego en el que se están creando verdaderas obras de arte y otras ideas que se proponen en las asambleas (un centro comercial, por ejemplo), donde también se encuentran momentos en los que los usuarios muestran actitudes de ayuda y colaboración con los compañeros.

Por último, la motivación que se explica en la intensidad, la persistencia y, también, la calidad de las conductas que tienen lugar dentro del club, tanto en el mundo virtual como en los puntos de encuentro de las asambleas.

\subsection{Resultados para la dimensión 3 de la investigación}

Esta tercera dimensión se enmarca dentro del cuestionario realizado a los familiares de los usuarios del club. Las preguntas se han analizado con una escala desde el 1 (totalmente en desacuerdo) a 4 (totalmente de acuerdo).

En la pregunta 1 el $100 \%$ de los familiares encuentran que el club mejora las relaciones con otras personas. De igual forma, en los ítems 2, 3 y 4 , manifiestan el $100 \%$ de acuerdo con que jugando a Minecraft se aprovecha el tiempo y se pueden desarrollar habilidades nuevas, además de que se divierten, tal y como muestran los gráficos. 
MINECRAFT EN EL APRENDIZAJE DE HABILIDADES SOCIALES PARA PERSONAS CON TRASTORNO DEL ESPECTRO DEL AUTISMO

FIGURA 5

Gráfico ítems 1, 2 y 3 del cuestionario

1. Minecraft mejora las relaciones con otras personas (14 respuestas)

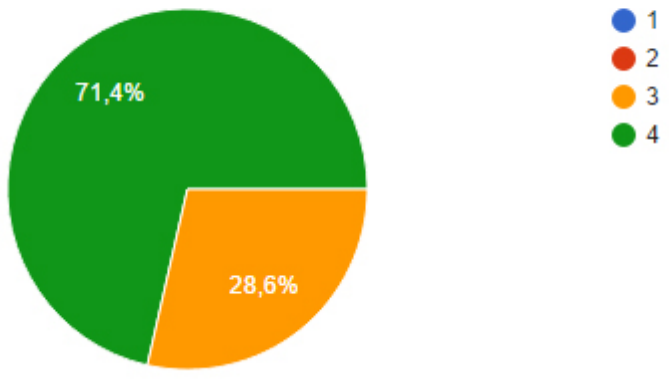

2. Jugando a Minecraft se aprovecha el tiempo (14 respuestas)
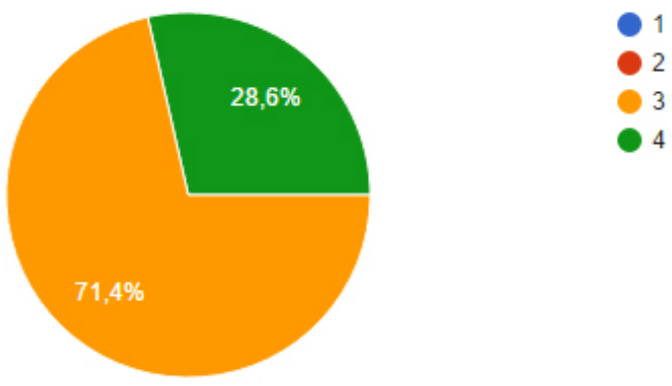

3. Minecraft ayuda a desarrollar habilidades nuevas (14 respuestas)
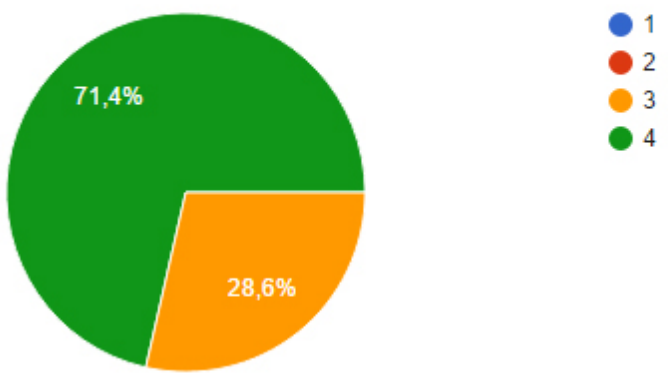
MINECRAFT EN EL APRENDIZAJE DE HABILIDADES SOCIALES PARA PERSONAS CON TRASTORNO DEL ESPECTRO DEL AUTISMO

FiguRA 6

Gráfico ítem 4 del cuestionario

\section{Cuando juega a Minecraft se divierte (14 respuestas)}

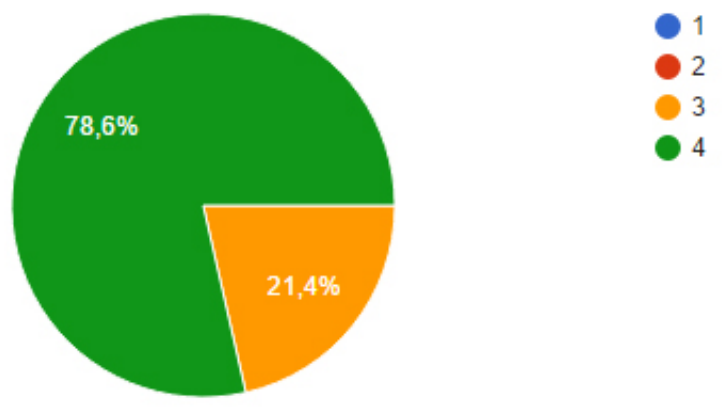

En los gráficos, se muestran los resultados para los ítems 5 y 6, pudiendo analizar que la mayor parte de los familiares están de acuerdo con que los usuarios inscritos en el club muestran mucho interés por los compañeros y compañeras del club y además hablan de Minecraft con otras personas ajenas al club, pudiendo comprobar también que existe un pequeño valor en cada uno (en el ítem 5 es insignificante y en el 6 encontramos un 14,3\% de respuestas que están en desacuerdo con esta afirmación). Valores no significantes para el estudio, pero sí para una evaluación del propio programa, con el fin de trabajar de forma más exhaustiva las vías de comunicación con otros compañeros o personas ajenas al club.

En el último gráfico de este cuestionario, la pregunta abierta, nos encontramos con que a la pregunta ¿Debería Club Minecraft formar parte de las terapias grupales para habilidades sociales?, tenemos un $21,4 \%$ que opinan que es divertido, un $42,9 \%$ que permite innovar y un 28,6 \% marca la opción 5 que se basaba en las otras dos opciones: es divertido y permite innovar. Solo un 7,1 \% opinan que ocasionalmente ayuda al resto de terapias y encontramos un $0 \%$ de respuestas que opinen que se pierde tiempo aplicando esta terapia. 
MINECRAFT EN EL APRENDIZAJE DE HABILIDADES SOCIALES PARA PERSONAS CON TRASTORNO DEL ESPECTRO DEL AUTISMO

\section{FIGURA 7}

Gráficos de los ítems 5, 6 y 7 del cuestionario

5. Muestra mucho interés por los compañeros y compañeras del club (14 respuestas)

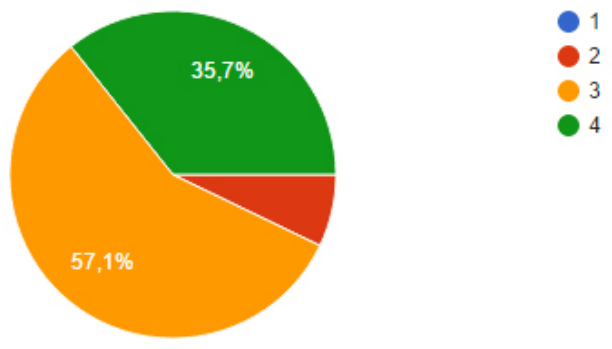

6. Habla de Minecraft con otras personas ajenas al club (14 respuestas)
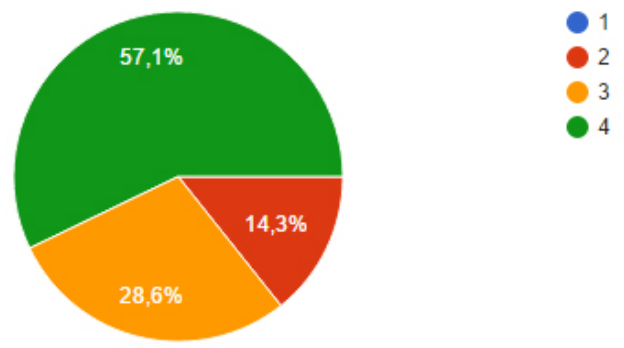

¿Debería Club Minecraft formar parte de las terapias grupales para habilidades sociales?

(14 respuestas)

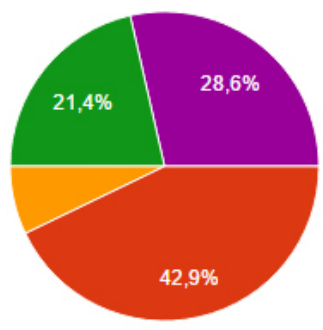

Se pierde tiempo aplicando esta terapia

Permite innovar

Ocasionalmente ayuda al resto de terapias

Es divertido

Opción 5 
Las familias muestran apoyo hacia el potencial terapéutico del videojuego y esto puede ser un factor fundamental en la motivación de los usuarios y en el desarrollo del proyecto por los profesionales, ya que se cuenta con su apoyo y confianza, tal y como demuestran en los resultados.

\section{CONCLUSIONES Y PROSPECTIVA}

Esta investigación ha querido estudiar el resultado de la aplicación del videojuego Minecraft como terapia para el aprendizaje de habilidades sociales en personas con TEA. Esta experiencia pionera e innovadora en el contexto español e internacional, se ha analizado a través de tres dimensiones basadas en los agentes implicados en esta investigación como son, en primer lugar, los usuarios del club; en segundo lugar, los profesionales involucrados, y, en tercer lugar, las familias de los usuarios.

Gracias al análisis de los instrumentos aplicados para cada una de estas dimensiones se han obtenido unos resultados que permiten la triangulación de los datos conseguidos, como recurso para la calidad de esta investigación, con el fin de potenciar las conclusiones que de estos datos se observan.

Siguiendo a Alonso y Gallego (2011) sobre cómo se adecuan los videojuegos al estudio de la inteligencia emocional y, en concreto, según se estudia en esta investigación, acerca de las habilidades sociales, es necesario partir de la base de que el videojuego tiene características y condiciones similares a las de los juegos y es por tanto que la primera conclusión que se extrae de estas tres dimensiones sería que el Club Minecraft tiene un enfoque lúdico donde los socios y socias del club están inmersos en un proceso de aprendizajes significativos mediante el juego, a través de actividades divertidas y amenas.

Siguiendo esta línea, se desprende la conclusión de que el videojuego Minecraft favorece la motivación por parte de los usuarios por pertenecer a un club, motivación que además les ha servido para la disposición al esfuerzo mantenido por conseguir las metas planteadas, el aprendizaje del funcionamiento del videojuego a pesar de encontrar dificultades en ello y que condiciona la capacidad de aprender, dotando a los aprendizajes de significado lógico y funcionalidad gracias a un interés común a otras personas.

Esta motivación se ha mantenido a lo largo de los meses donde, además, se ha afianzado el sentimiento de pertenencia a un grupo, acentuado en la aportación de ideas y mejoras por parte de los miembros y en la proliferación de las interacciones personales en las asambleas.

Por tanto, y apoyándonos en González y Quero (2013), para sintetizar estas conclusiones encontramos que, por un lado, la motivación en el empleo de videojuegos supone tres elementos fundamentales: la automotivación, el compromiso y la perseverancia. De esta forma, en cuanto al análisis de los intereses por Minecraft de los usuarios se destaca que existe automotivación, gracias al incremento de la dificultad del videojuego; existe compromiso, corroborado por la firma del 
contrato del club y el cumplimiento de las normas, y, por último, existe perseverancia, expuesta en el interés demostrado por los usuarios por pertenecer al club y, además, obtener las insignias de cada temporada.

Por último, nos encontramos con que el uso de Minecraft presenta una eficacia terapéutica desde la perspectiva de mejora en las relaciones sociales, aprovechamiento del tiempo y aprendizaje de habilidades nuevas, sin olvidar que el uso de la tecnología y en este caso del videojuego Minecraft debe ir unido, de forma inseparable, con una estructuración de normas y estrategias metodológicas. Este club permite aprovechar al máximo esta herramienta tecnológica como terapia para el aprendizaje de habilidades sociales en personas con TEA.

En definitiva, se puede concluir que el videojuego Minecraft posee un enfoque lúdico, que favorece la motivación mostrada por parte de los socios y socias del club y que conlleva una eficacia terapéutica por lo que puede ser utilizado como herramienta para los profesionales, tal y como demuestra este estudio, ya que permite el aprendizaje de habilidades sociales involucrando a los propios usuarios en el desarrollo del juego, gracias a los elementos de diversión y motivación que presenta, facilitando la adquisición de dichos aprendizajes sociales.

Finalmente, esta investigación abre una nueva línea de estudio enfocada en el desarrollo de terapias asistidas a través de videojuegos para personas con TEA. De esta forma, el videojuego Minecraft puede suponer una herramienta fundamental para futuras investigaciones donde, en colaboración con las familias, se pueda comprobar en qué momentos o qué circunstancias provocan frustración o alteran sus índices de ansiedad en situaciones de juego y, de esta forma, conocer más profundamente las conductas desencadenantes de ansiedad y plantear posibles estrategias para el control de esta y la autorregulación emocional.

\section{REFERENCIAS BIBLIOGRÁFICAS}

American Psychiatric Association. (2014). DSM-5. Manual diagnóstico y estadístico de los trastornos mentales. Editorial Médica Panamericana.

Aresti-Bartolome, N. y Garcia-Zapirain, B. (2014). Technologies as Support Tools for Persons with Autistic Spectrum Disorder: A Systematic Review. International Journal of Environmental Research and Public Health, 1660-4601. http://www.mdpi.com/1660$4601 / 11 / 8 / 7767$.

Cacheiro González, M. L.; Sánchez Romero, C. y González Lorenzo, J. M. (Coords.). (2015). Recursos tecnológicos en contextos educativos. Madrid: Universidad Nacional de Educación a Distancia.

Domínguez, M. C.; Medina, A. y Cacheiro, M. L. (Coords.). (2010). Investigación e Innovación de la Docencia Universitaria en el Espacio Europeo de Educación Superior. Madrid: Ramón Areces.

Ferguson, B. R.; Gillis, J. M. y Sevlever, M. (2013). A Brief Group Intervention Using Video Games to Teach Sportsmanship Skills to Children with Autism Spectrum Disorders. Child E Family Behavior Therapy, 35, 4. 293-306. http://www.tandfonline.com/doi/ful 1/10.1080/07317107.2013.846648. 
MINECRAFT EN EL APRENDIZAJE DE HABILIDADES SOCIALES PARA PERSONAS CON TRASTORNO DEL ESPECTRO DEL AUTISMO

Gallagher, C. (2015). Minecraft in the Classroom. Ideas, inspiration, and student projects for teachers. United States of America: Peachpit Press.

Garzotto, F.; Gelsomini, M.; Oliveto, L. y Valoriani, M. (2014). Motion-Based Touchless Interaction for ASD Children: a Case Study. AVI Proceedings of the 2014 International Working Conference on Advanced Visual Interfaces (pp. 117-120). http://www.academia. edu/6832738/Motion-based_Touchless_Interaction_for_ASD_Children_a_case_study.

González, J. M. y Quero, M. (2013). Arriesgar sin riesgos: videojuegos para la creatividad. Revista de Estudios de Juventud, 13, Madrid: INJUVE.

Jiménez Peñuela, J. R. (2010). Posibilidades educativas de las Tic para población infantil autista: buscadores y software especializado. @tic. revista d'innovació educativa, 5. http://dialnet.unirioja.es/descarga/articulo/3362212.pdf.

Lee, H. W. y Hyun, E. J. (2015). The Intelligent Robot Contents for Children with SpeechLanguage Disorder. Educational Technology \& Society, 18(3), 100-113. http://www. ifets.info/journals/18_3/8.pdf.

Lozano-Martínez, J. y Alcaraz-García, S. (2011). Software educativo para la enseñanza de competencias emocionales en alumnado con trastornos del espectro autista. Educación XX1. 14.2, 2011, 189-212. http://revistas.uned.es/index.php/educacionXx1/article/ view/250/206.

Méndez, L. y Del Moral, M. E. (2015). Investigación e innovación educativa con videojuegos. Electronic Journal of Research in Educational Psychology, 13 (2), 211-218. http://www. investigacion-psicopedagogica.org/revista/new/ContadorArticulo.php?1046.

Molyneux, L.; Vasudevan, K. y Gil de Zúñiga, H. (2015). Gaming Social Capital: Exploring Civic Value in Multiplayer Video Game. Journal of Computer Assisted Learning, 20, 381399. http://onlinelibrary.wiley.com/doi/10.1111/jcc4.12123/full.

Moral Pérez, M. E. y Fernández García, M. C. (2015). Videojuegos en las aulas: implicaciones de una innovación disruptiva para desarrollar las Inteligencias Múltiples. Revista Complutense de Educación, 26. Núm. Especial: TIC en Educación, 97-118. http://revistas. ucm.es/index.php/RCED/article/view/44763/45933.

Ojea, M. (2005). Trastornos generalizados del desarrollo: orientación educativa y atención en la diversidad. Santiago de Compostela: Tórculo.

Ojea, M. (2007). Autismo: entender, leer y hablar. Málaga: Editorial Aljibe.

Sáez López, J. M. (2011). Opiniones y práctica de los docentes respecto al uso pedagógico de las tecnologías de la información y la comunicación. Reid. Revista Electrónica de Investigación y Docencia, 5, 95-113. http://revistaselectronicas.ujaen.es/index.php/ $\mathrm{reid} /$ article/view/1065.

Sáez López, J. M. (2012). Valoración de la persistencia de los obstáculos relativos al uso de las tecnologías de la información y la comunicación en educación primaria. Educatio Siglo XXI: Revista de la Facultad de Educación, 30 (1), 253-274. http://revistas.um.es/ educatio/article/view/149231.

Sáez López, J. M., y Domínguez, C. (2013). Integración pedagógica de la aplicación Minecraft Edu en educación primaria: un estudio de caso. Píxel-Bit, Revista de Medios y Educación, 45, 95-110. http://dx.doi.org/10.12795/pixelbit.2014.i45.07.

Sáez-López, J. M.; Miller, J.; Vázquez-Cano, E. y Domínguez-Garrido, M. C. (2015). Exploring Application, Attitudes and Integration of Video Games: MinecraftEdu in Middle School. Educational Technology \& Society, 18 (3), 114-128. http://www.ifets.info/journals/18_3/9.pdf. 
Wijnhoven, L. A. M. W.; Creemers, D. H. M.; Engels, R. C. E. y Granic, I. (2015). The effect of the video game Mindlight on anxiety symptoms in children with an Autism Spectrum Disorder. BMC Psychiatry, 15, 138. http://link.springer.com/article/10.1186/s12888-0150522-x/fulltext.html.

\section{SiTIOS WEB}

AEVI. Asociación Española de Videojuegos. http://www.aevi.org.es/.

Asociación Desarrollo Autismo Albacete. https://www.autismoalbacete.org/. 